\title{
HI AND CONTINUUM STRUCTURE OF EXTERNAL GALAXIES COMPARED TO THE SITUATION IN THE GALAXY
}

\author{
Arnold H. Rots \\ Netherlands Foundation for Radio Astronomy \\ Dwingeloo, The Netherlands
}

Continuum observations of NGC 6946 strongly suggest the presence of an exponential non-thermal disk component. When applied to galactic $408 \mathrm{MH}$, observations, the scale length is found to be between 5 and $6 \mathrm{kpr}$. Continuing the parallel with NGC 6946, a total luminosity of $2 \times 10^{10} \mathrm{~L}_{\odot}$ is derived. Observations of the edge-on galaxies NGC 891 and 4631 show the existence of flattened halos with a steep spectrum around these galaxies. This is consistent with the constraints that are known for the galactic radio halo. On the basis of the radial distribution of $H I$ in the Galaxy and in M81 three regimes can be defined: a central one which is hyperdeficient in hydrogen; a middle one which is deficient in HI, but not necessarily in total hydrogen; and an outer one which is neither deficient in HI, nor in H. Density wave theory, combined with gas flow dynamics appear a powerful tool in interpreting the kinematical data. No rotation curve is yet known to become Keplerian in the outer parts. Many

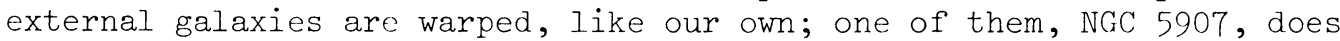
not have a visible companion.

\section{INTRODUCTION}

Since the previous IAU Symposium on the Galaxy (Galactic Radio Astronomy, 1973, Maroochydore) many new data have become available in the fields of galactic and extra-galactic radio astronomy. It is far from true, however, that a unifying picture has emerged, or that it could be put together in a paper like this one. I would just like to bring together some extragalactic examples and various aspects of galactic research.

For more detailed information and references to the existing literature the reader may be referred to the following reviews. For galactic continuum radiation to the ones by Price (1974b) and Baldwin (1977), for "HI and other tracers" in the Galaxy to those by Burton $(1976,1977)$, and to the reviews by Van der Kruit and Allen (1976, 1978) on external galaxies. 


\section{DISTRIBUTION OF CONTINUUM RADIATION}

\subsection{Radial distribution}

Price (1974a,b) showed that the non-thermal base disk component of the galactic radiation at low frequencies can be very well fitted by an exponential disk. I.e., a disk model in which the volume emissivity is proportional to exp $(-r / a)$, where $r$ is the galactocentric radius and a the scale length; he favoured a scale length of $6 \mathrm{kpc}$. Baldwin (1977), on the other hand, argued that a good fit can be obtained with a fairly uniform disk that rapidly falls off just inside the solar circle.

A suitable external galaxy for studying its non-thermal disk is the spiral system NGC 6946. Van der Kruit et al. (1977) obtained continuum distributions for this galaxy at $0.6,1.4$, and $5 \mathrm{GHz}$ with the Westerbork Synthesis Radio Telescope (WSRT). They conclude that the base disk component shows a nearly exponential behaviour with radius.

This is one more reason to look at the exponential models again. Fig. 1 shows the continuum radiation vs. galactic longitude profile derived by Green (1974) from her survey of the galactic plane at $408 \mathrm{MHz}$, done with the Molonglo Cross antenna. The heavy curve shows the profile that would be expected from an exponential disk with a scale length of $5 \mathrm{kpc}$. If one takes the liberty of having some doubts about the accuracy with which one can determine the zero level of these low frequency surveys, it is possible to get a very good fit for an exponential model with a scale length of $6 \mathrm{kpc}$ as well. The broken curve represents such a model, assuming that the true zero level is $25 \mathrm{~K}$ below Green's.

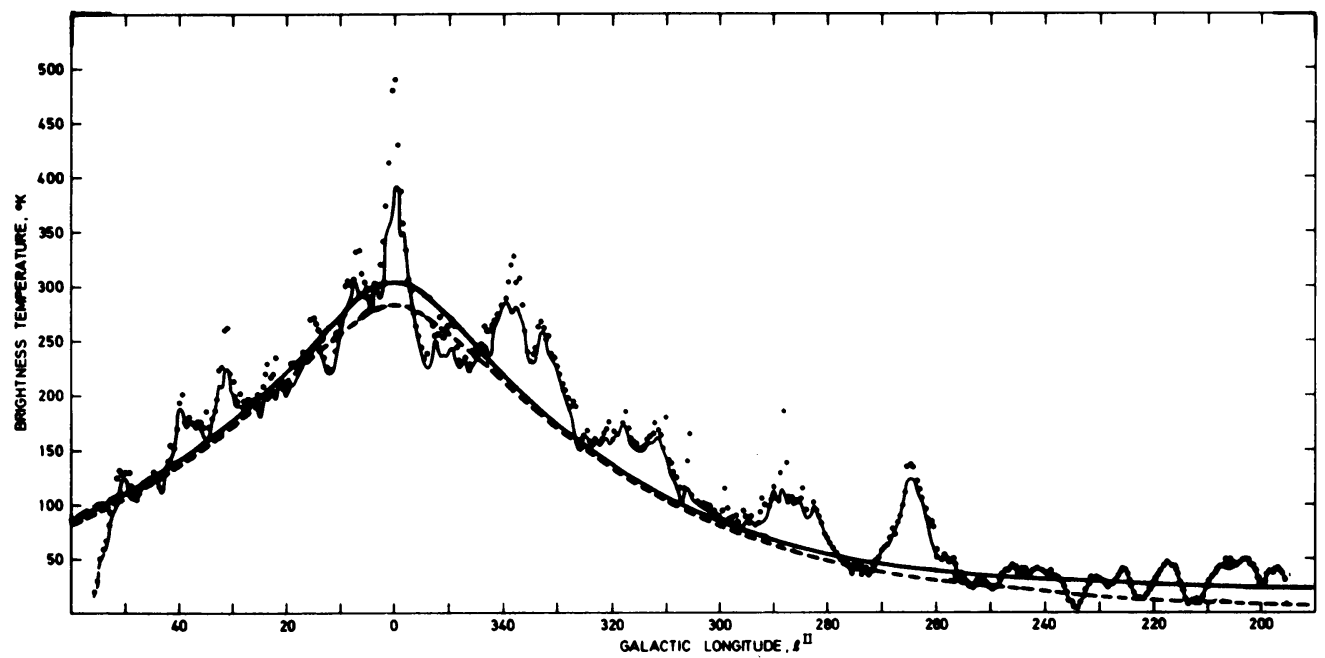

Fig. 1. Brightness temperature at $408 \mathrm{MHz}$ along the galactic plane according to Green (1974). The smooth and broken line are exponential models for the disk with scale lengths of, resp., 5 and $6 \mathrm{kpc}$ (see text).

Both fits (and especially the latter) are in my opinion quite satisfactory and certainly no worse than Baldwin's model. For a determination of the 
exact scale length one need to separate the disk radiation from the nondisk components in the profile. This is especially difficult in the center and anti-center directions. We can conclude that if the non-thermal base disk has an exponential brightness distribution, the scale length at $408 \mathrm{MHz}$ must be between 5 and $6 \mathrm{kpc}$ (assuming the distance to the galactic center to be $10 \mathrm{kpc}$ ).

Comparison with the light distribution proves to be very interesting. Van der Kruit et al. (1977) found that the scale length of the non-thermal disk in NGC 6946 is very close to the scale length of the exponential disk representing the distribution of visible light as derived by Ables (1971). A similar correspondence for M51 had been pointed out by Allen (1975). If this close correlation between the two scale lengths could be well established it would provide an independent method for estimating the total luminosity of the Galaxy (Lpg) from:

i. The scale length of the non-thermal disk (a).

ii. The distance of the galactic center $\left(R_{O}\right)$.

iii. The local optical surface brightness in the solar neighbourhood $\left(\sigma_{\odot}\left(L_{\odot} p c^{-2}\right)\right)$.

Lpg can then be expressed as: $L_{p g}=b \times \sigma_{\odot}\left(L_{\odot}\right)$. Integrating out to a radius of $30 \mathrm{kpc}$ one obtains $\mathrm{b}=1.15 \times 10^{9}$ for $\mathrm{a}=5.5 \mathrm{kpc}$ and $\mathrm{R}_{\mathrm{O}}=10 \mathrm{kpc}$. The uncertainty in a is not important since it results in less than one percent variation in $\mathrm{L}_{\mathrm{pg}}$. For $\mathrm{R}_{\mathrm{O}}=8.5 \mathrm{kpc}$ one obtains $\mathrm{b}=0.83 \times 10^{9}$. Hence we shall adopt $\mathrm{b}=10^{9}\left( \pm 15 \%\right.$ due to the uncertainty in $\left.\mathrm{R}_{0}\right)$. Additional errors arise from:

i. The uncertainty in $\sigma_{\odot}$.

ii. Neglecting the light contribution from the spiral arms.

iii. Neglecting the light contribution from the nuclear bulge. The total uncertainty may well be a factor 2 . The result and two relevant ratios are shown in the table. The value for $\sigma_{\odot}\left(21 \mathrm{~L}_{\odot} \mathrm{pc} c^{-2}\right)$ has been taken from Oort (1965); the total mass from Innanen (1973); and the HI mass from Kerr and Westerhout (1965) and Burton (1976). The M/L averages for the two morphological types $\mathrm{Sb}$ and $\mathrm{Sbc}$ are from Roberts (1975). One should be warned that the higher weight that is usually attached to $\mathrm{MHI}_{\mathrm{H}} \mathrm{L}$ (since it is a distance independent parameter for external galaxies) is not justified in this particular case. The HI mass of the Galaxy is uncertain because of uncertainties in precisely that parameter: the distance. It seems fair to conclude that the present determination is consistent with the notion of our Galaxy being of morphological type Sbc or slightly earlier.

\begin{tabular}{llcll}
\hline & & Galaxy & $\langle\mathrm{Sb}\rangle$ & $\langle$ Sbc \\
\hline $\mathrm{M}_{\mathrm{pg}}$ & $(\operatorname{mag})$ & -20.4 & & \\
$\mathrm{~L}_{\mathrm{pg}}$ & $\left(10^{9} \mathrm{~L}_{\odot}\right)$ & 20 & & \\
$\mathrm{M}_{\mathrm{T}}$ & $\left(10^{9} \mathrm{M}_{\odot}\right)$ & 110 & & \\
$\mathrm{M}_{\mathrm{HI}}$ & $\left(10^{9} \mathrm{M}_{\odot}\right)$ & 3 & & \\
$\mathrm{M}_{\mathrm{T}} / \mathrm{L}$ & $\left(\mathrm{M}_{\odot} / \mathrm{L}_{\odot}\right)$ & 5.5 & $6.4( \pm 2.1)$ & $6.0( \pm 1.2)$ \\
$\mathrm{M}_{\mathrm{HI}} / \mathrm{L}$ & $\left(\mathrm{M}_{\odot} / \mathrm{L}_{\odot}\right)$ & 0.15 & $0.17( \pm 0.03)$ & $0.22( \pm 0.03)$ \\
\hline
\end{tabular}


2.2. z-distribution

I want to emphasize that the use of the word "halo" does not necessarily imply that the feature which it refers to has a spheroidal shape.

In recent years some new data have become available on halos of external galaxies. Allen et al. (1978) show the existence of a flattened halo around the edge-on galaxy NGC 891. At $1.4 \mathrm{GHz}$ the continuum radiation extends at least to a height of $6 \mathrm{kpc}$ above the plane of the galaxy, assuming a distance of $14 \mathrm{Mpc}$. Observations of NGC 4631 at the same frequency by Ekers and Sancisi (1977) show a vertical extent of at least $7 \mathrm{kpc}$, at an assumed distance of $5.2 \mathrm{Mpc}$. The axial ratio of the halo component is larger for NGC 4631 than for NGC 891. In both cases there are strong indications that the spectrum steepens considerably with increasing height above the plane: in the case of NGC 891 from 0.6 at heights up to $\mathrm{z}=2.5 \mathrm{kpc}$ to 1.0 or 1.5 at $\mathrm{z}=4 \mathrm{kpc}$.

These data are consistent with statements by Price (1974b) and Baldwin (1977) that the existence of a radio halo with a steep spectrum around our Galaxy is not precluded by low-frequency observations, and with the steep spectrum halo component derived by Webster (1975). One should note, however, that such a halo may very likely not be spheroidal. A thorough investigation of the change of spectral index with $\mathrm{z}$ would be very interesting, but requires higher sensitivity and/or lower observing frequencies then the above mentioned WSRT observations offer.

\subsection{Origin of the relativistic electrons.}

The question of the origin of the relativistic electrons responsible for the large scale non-thermal radiation of galaxies is actually beyond the scope of this paper. Two short remarks can be made, however.

It is important to note that, apparently, the non-thermal disk (producing the bulk of the non-thermal radiation) mimics the Population II distribution. Whether the supernovae are distributed in the same manner is not entirely clear. The reader be referred to the short discussion on this subject by Van der Kruit et al. (1977).

In trying to understand the mechanisms that are at work, various other tracers may hold important clues. Galactic research has an important advantage here over extra-galactic research. For reasons of sensitivity and resolution several constituents (e.g. $\gamma$-radiation, molecular radiation) cannot be observed with enough detail in external galaxies.

\section{THE DISTRIBUTION AND KINEMATICS OF NEUTRAL ATOMIC HYDROGEN}

\subsection{Radial distribution}

The two most striking features in the radial distributions of $\mathrm{HI}$ in galaxies which have been known for many years, are the deficiency of HI in the central regions of most galaxies, and the fact that the HI seems to extend to much larger radii than all other tracers.

The radial HI distributions for the Galaxy (see Burton, 1976) and M81 (see Fig. 2 which is taken from Rots, 1975) both show a central 


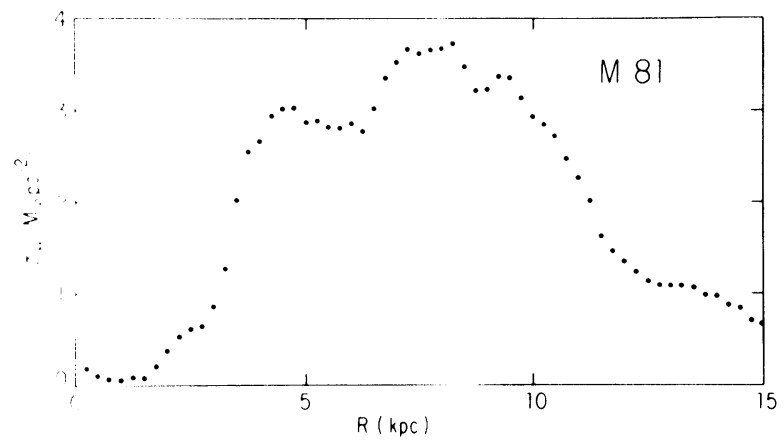

Fig. 2. HI surface density as a function of radius in M81 (from Rots, 1975).

deficiency, a steep increase, a plateau of rather constant surface density, and a sudden drop followed by a gradual decline as radius increases. It is remarkable that in both cases the two discontinuities at either side of the plateau region seem to occur at about the same radii as, resp., the inner Lindblad resonance and corotation. However unfortunately located our vantage point in the Galaxy may be, it is here that we have the best observational conditions (in terms of sensitivity and resolution) for studying these resonance regions in detail.

As for the alleged large extent of the HI: Burton (1977, see Fig.3) shows that beyond $6 \mathrm{kpc}$ the total hydrogen surface density (including molecular hydrogen) rather closely follows the total mass distribution as determined by Innanen (1973). The determination of the $\mathrm{H}_{2}$ surface densities is not very certain, but for the Galaxy as well as for M81 the assertion seems valid that the relative HI surface density -after a gradual increase- remains more or less constant beyond the corotation region. One should bear in mind, however, that there still is considerable uncertainty in the mass models and in the galactic radial distribution of HI. On the other hand, Bosma (1978) finds the same effect in a

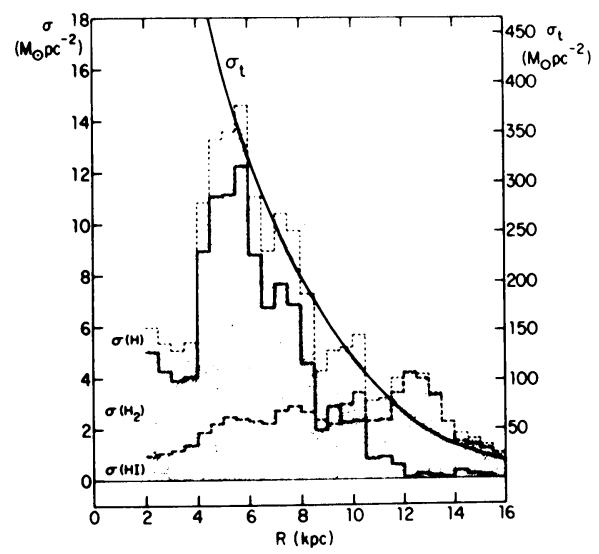

Fig. 3. Surface densities of $\mathrm{HI}, \mathrm{H}_{2}, \mathrm{HI}+\mathrm{H}_{2}$, and total mass (Innanen,1973) in the Galaxy, from Burton (1977). 
sample of 14 galaxies.

Thus one may tentatively reformulate the "striking features" mentioned at the beginning of this section in terms of the relative HI surface density $(\mu \mathrm{HI}$ ) and discern three areas: (in order of decreasing radius)

i. In the outer regime $\mu_{\mathrm{HI}}$ is more or less constant. Most of the hydrogen is in the form of HI. There is no deficiency of either $\mathrm{H}$ or $\mathrm{HI}$.

ii. In the middle regime there is an increasing deficiency of HI with decreasing radius (i.e.: $\mu_{\mathrm{HI}}$ decreases). Since the fraction of $\mathrm{H}_{2}$ increases rapidly in this region there may not be a deficiency of $\mathrm{H}$ ( $i . e .: \mu_{\mathrm{H}}$ may still remain constant)

iii. In the central regime there is a (hyper-)deficiency of both $\mathrm{HI}$ and $\mathrm{H} . \mu_{\mathrm{HI}}$ and $\mu_{\mathrm{H}}$ quickly drop to (near) zero.

The central regime is obviously dominated by the nuclear bulge; studies of elliptical galaxies may help to understand this region. It is interesting to speculate whether or not the rough coincidence of the regime boundaries and the density-wave-theory resonances is significant.

\subsection{Kinematics}

Various types of kinematical data for many galaxies are available. We may refer to Van der Kruit and Allen (1978) for a review of these, and to Bosma (1978) for additional data. As far as the dependence on morphological type is concerned, the available data seem to support the notion (Roberts and Rots, 1973) that earlier type galaxies do show a higher degree of mass concentration toward the center.

Intimately related to the problem of determining rotation curves is the matter of spiral structure, if only because the kinematical effects of the latter confuse determination of the former. This has been worked out rather convincingly by Visser (1978) for the case of M81 (see Fig. 4). He corrected the velocity field for streaming motions and removed in that manner the steep decline around $8 \mathrm{kpc}$ that caused Rots (1975) difficulties in fitting a mass model. It should be remarked, however, that in a case like this one, where a reasonably satisfactory rotation curve and mass model have been derived, yet another problem appears: deviations from the crucial assumption of axial symmetry (for M81 a north-south asymmetry in the velocity field; c.f. Rots, 1974, 1975).

This paper will not deal with spiral structure specifically, except to note that models based on density wave theory and gas flow dynamics (including shocks) seem quite capable of reproducing the observed kinematics satisfactorily. Such detailed models have been worked out for the Galaxy by Simonson (1976) and for M81 by Visser (1978). A basic difference in the approaches of these two authors is that Visser corrected the rotational data iteratively for gas flows linked to the spiral structure, while Simonson kept his rotation curve (Schmidt, 1965) fixed.

As far as the rotational data from HI observations in the outer parts of galaxies is concerned, it is fair to say that no rotation curve has yet been derived which turns Keplerian before radio telescopes run out of signal. 


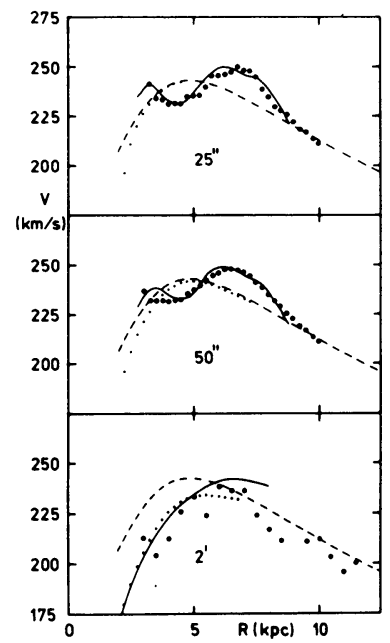

Fig. 4. Rotational data for M81 at different resolutions, from Visser (1978). Filled circles: observed velocities; broken curve: axisymmetric model; full curve: model plus streaming motions; dotted curve: axisymmetric model distorted by the beam.

\subsection{HI in the outer parts of galaxies}

It is well known that although the HI is generally concentrated in well defined flat disks, the outer parts of many galaxies greatly deviate from the planes of such disks. The Galaxy's HI disk is known to be warped in the outer regions, and several people have tried to explain this phenomenon by gravitational interaction with the Magellanic Clouds (see e.g. Hunter and Toomre, 1969). M31 is now known to be warped (Roberts and Whitehurst, 1975); M33 has a very strong warp (Rogstad et al., 1976); M83 too (Rogstad et al., 1974); IC 342 is conceivably warped (Rogstad et al., 1973); M81 shows HI almost everywhere and it seems unlikely that it would be confined to one plane; and there are many other examples. Quite interesting is the study of Sancisi (1976) who found warps in four out of five edge-on systems. Interesting because of the statistics, but also because one of the warped systems, NGC 5907, does not have a visible companion. This case should renew the interest in alternative warping mechanisms, as has been pointed out by Burton (1976).

The case of M81 that was just mentioned, does not have a proper, clean warp, but actually belongs in the realm of bridges, tails, and Magellanic Streams. Several years ago Toomre and Toomre (1972) produced the first successful gravitational interaction models. Some models have recently proved to be consistent with HI observations (with their added kinematical information): NGC 4038/9 by Van der Hulst (1977), NGC 3628/7 by Rots (1978). In our own Galaxy, however, we are faced with a warp, a Magellanic Stream, and the fact that we are not "outside observers", which seriously restricts our knowledge of the geometry and kinematics. And this yields another parallel with M81: both systems are extremely complicated as far as fitting gravitational interaction models is concerned. 


\section{REFERENCES}

Ables, H.D.: 1971, Publ. U.S. Naval Obs., Ser. II, Vol. XX, Part IV. Allen, R.J.: 1975, in L. Weliachew (ed.), "La Dynamique des Galaxies Spirales" (CNRS), p. 157.

Allen, R.J., Baldwin, J.E., Sancisi, R.: 1978, Astron. Astrophys. 62, p. 397 .

Baldwin, J.E.: 1977, in C.E. Fichtel and F.W. Stecker (eds.), "Structure and Content of the Galaxy and Galactic Gamma Rays" (NASA), p. 189.

Bosma, A.: 1978, Dissertation, University of Groningen.

Burton, W.B.: 1976, Ann. Rev. Astron. Astrophys. 14, p. 275.

Burton, W.B.: 1977, in C.E. Fichtel and F.W. Stecker (eds.), "The Structure and Content of the Galaxy and Galactic Gamma Rays" (NASA), p.163.

Ekers, R.D., Sancisi, R.: 1977, Astron. Astrophys. 54, p. 973.

Green, A.J.: 1974, Astron. Astrophys. Suppl. Ser. 18, p. 267.

Hulst, J.M. van der: 1977, Dissertation, University of Groningen.

Hunter, C., Toomre, A.: 1969, Astrophys. J. 155, p. 747.

Innanen, K.A.: 1973, Astrophys. Space Sci. 22, p. 393.

Kerr, F.J., Westerhout, G.: 1965, in A. Blaauw and M. Schmidt (eds.), "Galactic Structure", Stars and Stellar Systems V, p. 167.

Kruit, P.C. van der, Allen, R.J.: 1976, Ann. Rev. Astron. Astrophys. 14, p. 417 .

Kruit, P.C. van der, Allen, R.J.: 1978, Ann. Rev. Astron. Astrophys. 16, in press.

Kruit, P.C. van der, Allen, R.J., Rots, A.H.:: 1977, Astron. Astrophys. 55, p. 421.

Oort, J.H.: 1965, in A. Blaauw and M. Schmidt (eds.), "Galactic Structure", Stars and Stellar Systems V, p. 455.

Price, R.M.: 1974a, Astron. Astrophys. 33, p. 33.

Price, R.M.: 1974b, in F.J. Kerr and S.C. Simonson (eds.), "Galactic Radio Astronomy", I.A.U. Symp. No. 60, p. 637.

Roberts, M.S.: 1975, in A. Sandage, M. Sandage and J. Kristian (eds.), "Galaxies and the Universe", Stars and Stellar Systems IX, p. 309.

Roberts, M.S., Rots, A.H.: 1973, Astron. Astrophys. 26, p. 483.

Roberts, M.S., Whitehurst, R.N.: 1975, Astrophys. J. 201, p. 327.

Rogstad, D.H., Lockhart, I.A., Wright, M.C.H.: 1974, Astrophys. J. 195, p. 309 .

Rogstad, D.H., Shostak, G.S., Rots, A.H.: 1973, Astron. Astrophys. 22, p. 111.

Rogstad, D.H., Wright, M.C.H., Lockhart, I.A.: 1976, Astrophys. J. 204 , p. 703 .

Rots, A.H.: 1974, Dissertation, University of Groningen.

Rots, A.H.: 1975, Astron. Astrophys. 45, p. 43.

Rots, A.H.: 1978, Astron. J. 83, p. 219.

Sancisi, R.: 1976, Astron. Astrophys. 53, p. 159.

Schmidt, M.: 1965, in A. Blaauw and M. Schmidt (eds.), "Galactic Structure", Stars and Stellar Systems V, p. 513.

Simonson, S.C.: 1976, Astron. Astrophys. 46, p. 261.

Toomre, A., Toomre, J.: 1972, Astrophys. J. 178, p. 623.

Visser, H.C.D.: 1978, Dissertation, University of Groningen.

Webster, A.: 1975, Monthly Notices Roy. Astron. Soc. 171, p. 243. 


\section{DISCUSSION}

Stecker: You mention the interesting possibility that the non-thermal emission in the Galaxy mimics the Population II distribution. If we assume that this reflects the distribution of sources of cosmic-ray electrons and of cosmic-ray nuclei as well, we would expect that the galactic nucleus would be much brighter in $\gamma$-rays than is observed. This may mean either that the sources of cosmic-ray electrons in our Galaxy have a different distribution than the sources of cosmic-ray nucleons (which seem to reflect the Population I distribution) or else the sources of cosmic-ray nucleons and electrons in the disk are the same (supernovae or pulsars) and there is an additional source (or sources) of cosmicrays in the galactic nucleus. This later possibility seems more attractive to me.

Wielebinski: The spectral-index increase of nonthermal emission away from the planes of NGC 881 and NGC 4631 is certainly real. This increase gives us the lifetimes of relativistic electrons. We should go back and try to determine the exact spectrum of the nonthermal emission in the Galaxy, to put limits on lifetimes of our cosmic rays. The measurements will not be easy, but should be considered a challenge.

de Vaucouleurs: Your observation that the mean radial distribution of the radio continuum emission in the disks of spirals is exponential with essentially the same scale length as the optical emission is most significant. However, the radio spectral index varies with radius and so does the optical color index. So which radio wavelength should be compared with which optical wavelength?

Rots: In NGC 6946 there is a variation of spectral index with radius in the sense that the spectrum steepens with increasing distance from the center. However, this can be entirely accounted for by the distributed thermal radiation in the inner parts of the disk. Thus the answer is that one should use a low frequency; $408 \mathrm{MHz}$ seems to be adequate.

Heiles: It seems to me that the facts that (1) the distributions of nonthermal and thermal (HII region) radio continuum have different shapes in any one galaxy, (2) that the relation between these shapes is different for M33, and (3) that HII regions are heated by hot, massive stars which become type II supernovae, show that at least some relativistic electrons are produced by processes other than type II supernovae. But, since type I supernovae also produce relativistic electrons, we cannot then conclude that processes other than supernovae are necessary.

Lockman: I do not agree that your conclusion necessarily follows from the facts you stated.

Heiles: I do not agree that my conclusion does not necessarily follow from the facts I stated. 
Oort: With regard to the possibility of producing relativistic electrons in other ways than by supernovae, it should be pointed out that there is now plenty of evidence in radio galaxies that high-energy particles can be produced in shock regions not connected with supernovae.

Bok: So far in this Symposium we have ignored the Magellanic Clouds. For example, we have not mentioned yet that different processes of star formation are at work in our Galaxy and in the Magellanic Clouds. In our Galaxy, star formation is apparently associated with low temperature carbon monoxide and molecular hydrogen. However, at a distance of 60,000 parsec from our Sun there are the two Magellanic Clouds in which star formation is at work on a very big scale, apparently mostly without benefit of large amounts of cold cosmic dust. Evidently excessive amounts of neutral atomic hydrogen are present in the Magellanic Clouds at many positions where star formation is actively in progress. Let us not ignore the Magellanic Clouds whenever they offer to tell us something of interest for consideration in studies of our own Galaxy. May I suggest that everyone who works on the large scale structure of our Galaxy place a picture of the Large or Small Magellanic Cloud on the walls of her or his office. Please bear in mind that the Clouds are at distances one-tenth of the distance to Messier 31! 